\title{
Single-incision laparoscopic surgery of cesarean scar ectopic pregnancy: a case report and review of literature
}

\begin{abstract}
Background: Cesarean scar ectopic pregnancy (CSEP) is the rarest location for ectopic pregnancy; with implantation within the cesarean scar defect (CSD) of the uterus. The primary pathology stems from wound poor healing, resulting in focal thinning of the uterine scar; predisposing the site for gestational sac implantation. Current CSEP treatment approaches include methotrexate (MTX), bilateral uterine artery embolization, dilation and curettage (D\&C), and open or laparoscopic surgical repair. We report a case of CSEP diagnosed via ultrasound and managed with methotrexate, (D\&C) with single-incision laparoscopic surgery (SILS) resection of the CSEP.

Case: 38-year-old Gravida 4, Para 3003 at 7-4/7weeks presented with intermittent bleeding and passage of clots. Bedside transvaginal ultrasound showed CSEP with detectable fetal heart tones. B-hCG value was $>30,000 \mathrm{mIU} / \mathrm{mL}$. Obstetrical history included a normal spontaneous vaginal delivery followed by two cesarean deliveries. For this case the patient opted for diagnostic hysteroscopy, suction D\&C, with SILS robotic resection of the CSEP, and umbilical hernia repair. The patient had an unremarkable recovery course and was discharged from the hospital on postoperative day 1, with plan to follow up weekly for quantitative B-hCG levels.

Conclusion: CSEP presents challenges for clinicians as there is conflicting data on the best mode of treatment. Our case presents a SILS surgical approach in combination with hysteroscopy for improved visualization. The authors have found this technique to be effective, but through review of the literature endorse the 3D-MESIA protocol alternative.

Keywords: 3D- MESIA, cesarean, cesarean scar defect, cesarean scar ectopic, cesarean scar ectopic pregnancy, cesarean scar pregnancy, diagnostic hysteroscopy, ectopic, fetal intracardiac potassium chloride injection, pregnancy, laparoendoscopic single-site surgery, methotrexate, uterine artery embolization, sonography directed-in situs aspiration sequential therapy based on the pregnancy sac three-dimensional (3d) conformation analysis (3d-mesia), residual myometrial thickness, robotic-assisted laparoscopy, singleincision laparoscopic surgery, uterine niche
\end{abstract}

Volume 9 Issue 3 - 2018

Shadi Rezai, ${ }^{1,6}$ Alexander C Hughes, ${ }^{2}$ Neil D Patel, ${ }^{2}$ Elise Bardawi, ${ }^{1,6}$ Yiming Zhang, ${ }^{3}$ Ninad M Patil, ${ }^{4}$ Cassandra E Henderson, ${ }^{5}$ Xiaoming Guan $^{6}$

'Department of Obstetrics and Gynecology, Southern California Kaiser Permanente, USA

${ }^{2}$ St George's University, School of Medicine, Grenada

${ }^{3}$ Division of Reproductive Medicine, Jinan Central Hospital Group, China

${ }^{4}$ Department of Pathology \& Immunology, Baylor College of Medicine, USA

${ }^{5}$ Maternal and Fetal Medicine, Department of Obstetrics and Gynecology, Lincoln Medical and Mental Health Center, USA ${ }^{6}$ Division of Minimally Invasive Gynecologic Surgery, Department of Obstetrics and Gynecology, Baylor College of Medicine, USA

Correspondence: Xiaoming Guan MD PhD, Section Chief and Fellowship Director, Division of Minimally Invasive Gynecologic Surgery, Department of Obstetrics and Gynecology, Baylor College of Medicine, 665I Main Street, I0th Floor, Houston, Texas, 77030, USA, Tel (832) 826-7464, Fax (832) 825-9349, Email rezsha@sgu.edu, Cassandra.henderson@nychhc.org

Received: March 24, 2018| Published: May 22, 2018
Abbreviations: CSD, cesarean scar defect; CSEP, cesarean scar ectopic pregnancy; CSP, cesarean scar pregnancy; LESS, laparoendoscopic single-site surgery; MTX, methotrexate; UAE, uterine artery embolization; RMT, residual myometrial thickness; SILS, single-incision laparoscopic surgery

\section{Background}

Cesarean scar ectopic pregnancy (CSEP) is the rarest location for ectopic pregnancy (incidence of $1: 1,800$ to $1: 1,216$ ) ${ }^{1,2}$ with implantation in the cesarean scar defect (CSD) of the uterus, ${ }^{3,4}$ There are two types of CSEP; Type 1 has progression toward the uterine cavity; Type 2 is deep within the myometrium. ${ }^{5}$ The primary pathology stems from poor wound healing, resulting in focal thinning of the uterine scar or migration of the embryo through a microscopic fistula wedge defect exposing the site for implantation..${ }^{6,7}$ Additional complications include rupture, hemorrhage, and uterine rupture due to weakness of the cesarean scar..$^{8-10}$

Ultrasound is the preferred diagnostic method for CSEP, with diagnostic criteria set as: a. Diagnosis of an empty uterine cavity

b. Diagnosis of an empty cervical canal

c. Development of the sac in the anterior isthmic segment

d. Circumferential flow using color Doppler

Absent or diminished myometrial thickness between the sac and maternal bladder. ${ }^{11,12}$

Since CSEP is so rare, no standard treatment exists - current approaches include methotrexate (MTX), bilateral uterine artery embolization, dilation and curettage (D\&C), and open or laparoscopic surgical repair. ${ }^{6,9,13}$ However, without surgical repair, the risk of recurrent CSEP still remains. ${ }^{14}$

We report a case of CSEP diagnosed via ultrasound and managed with MTX, D\&C and single-incision laparoscopic surgery (SILS) resection of the CSEP.

\section{Presentation of the case}

A 38-year-old, gravida 4 para 3003 at 7 weeks and 4 days gestation by last menstrual period presented with intermittent bleeding, passage 
of clots, and morning sickness. Obstetrical history included a normal spontaneous vaginal delivery followed by two cesarean deliveries, the last one being 2 years prior. Patient reported sulfa drug allergy, prior smoking status, and Rh positive status; period her body-mass index was $26.73 \mathrm{~kg} / \mathrm{m}^{2}$. she was hemodynamically stable.

Bedside transabdominal ultrasound confirmed a gestational sac, fetal pole, heart tones, and heart rate within a CSEP defect. B-hCG was $>30,000 \mathrm{mIU} / \mathrm{ml}$, with all other laboratory values within normal limits.

Treatment options were discussed with the patient, including MTX administration (systemic vs combined systemic and local intragestational), and hysteroscopy with SILS laparoscopic resection of CSEP.

Surgical evaluation revealed an 8 -week size, anteverted, mobile uterus with no additional masses; cervix was dilated to fingertip only. A $25 \mathrm{~mm}$ skin incision was made in the umbilicus and a GelPOINT Mini advanced access single-site laparoscopy device was inserted. Abdominal insufflation revealed vesico-uterine adhesions limiting immediate visualization of the ectopic pregnancy. Due to poor visibility, intraoperative hysteroscopy was performed which revealed a gestational sac at the lower segment of the uterus, adherent to the anterior uterine wall approximately $3-4 \mathrm{~cm}$ superior to the external cervical os. The RUMI manipulator was placed in the vagina without use of the uterine balloon. The vesico-uterine adhesions were lysed using the Harmonic scalpel and the ectopic pregnancy was exposed within the middle of the scar. Vasopressin was used for hemostasis and the Harmonic was used to incise this area; amniotic fluid with products of conception (POC) was noted and removed (Figure 1). Suction and graspers were used to remove as much POC tissue as possible. The RUMI manipulator was removed, and the endometrial tip was placed to ensure that the os was not closed during the repair The uterine defect was closed in 3 layers using V-loc sutures (Figure 2). While maintaining observation with the abdominal camera, a suction D\&C was performed. The diagnostic hysteroscopy, suction D\&C, and SILS robotic resection of the CSEP was performed with an estimated blood loss of $30 \mathrm{ml}$.

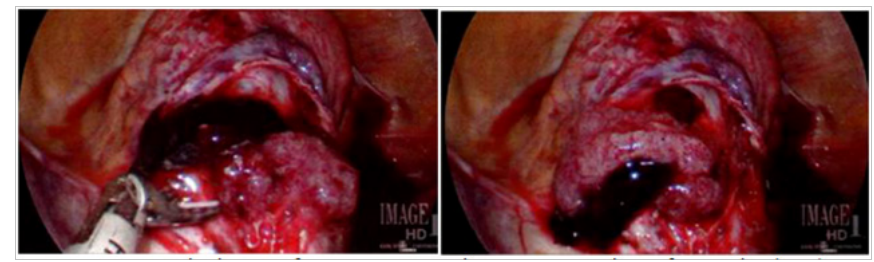

Figure I Intraoperative images of cesarean scar ectopic pregnancy; products of conception (POC) were noted and removed.

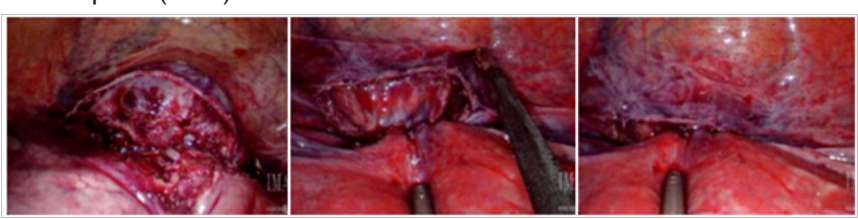

Figure 2 The uterine defect was closed in 3 layers using V-loc sutures.

Pathologic examination confirmed the products of the CSEP (first trimester chorionic villi) as well as fragments of adjacent decidua and implantation site (Figure 3).

The patient had an unremarkable recovery course and was discharged on postoperative day 1 with a plan for weekly follow-up quantitative B-hCG levels. Future pregnancy prevention methods were discussed; however the patient opted for abstinence and condoms.

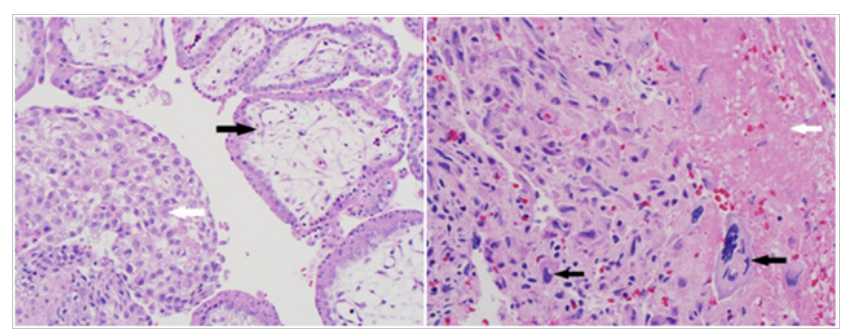

Figure 3 A \& B Pathology slides: A. Chorionic villi (black arrow) with nucleated fetal red cells consistent with first trimester, and extravillous trophoblast (white arrow); H\&E stain. B. Implantation-site trophoblast (black arrow), and fibrinoid (white arrow); (H\&E stain).

\section{Discussion}

CSEP is the rarest form of ectopic pregnancy with implantation occurring in the CSD. ${ }^{3}$ The embryo implants within the uterine wall because of thinning of the uterine scar, increasing the risk of uterine rupture. ${ }^{7}$ Most ectopic pregnancies result in spontaneous abortion, however, given the 'normal' position within the uterus, asymptomatic patients report no concern. In a recent review of CSEP, MaheuxLacroix et al. ${ }^{15}$ found that less than half of the cases reviewed had a previous live birth where majority required a cesarean hysterectomy. They concluded that expectant management exposes women to a higher risk of life-threatening hemorrhage and hysterectomy. ${ }^{15,16}$ Therefore, termination, evacuation and laparoscopic repair of the uterine scar is the preferred treatment ${ }^{17}$ however a gold standard has not been established. ${ }^{8,9,14,18}$ For cases presenting with extensive adhesions and limited visibility, intraoperative hysteroscopy has been successfully used for scar and implantation isolation. ${ }^{15}$

A new protocol called 3D-MESIA was recently introduced by Wang et al. ${ }^{14}$ proposing a systematic protocol for the management of CSEP. 3D-MESIA utilizes methotrexate (MTX), uterine artery embolization (UAE), and sonography directed-in situs aspiration (SIA), with multi-dimensional conformational analysis (3D) (Table 1). ${ }^{19}$ It is postulated to be easier, safer and more efficacious compared to previous therapy options. ${ }^{19} \mathrm{~A}$ recent study compares six different therapeutic approaches (3D-MESIA, systemic MTX injection, uterine artery chemoembolization/UAE with systemic MTX injection, uterine curettage after systemic MTX injection, uterine curettage after UAE, uterine curettage directly) for endogenous and exogenous CSEP. ${ }^{19}$ The 3D-MESIA approach was shown to be superior compared to the other five treatments examined. ${ }^{19}$ The greatest benefits noted were less intraoperative blood loss, reduced B-hCG clearance time, and shorter lesion absorption time of exogenous CSEP. ${ }^{19}$ 3D-MESIA failure would ultimately require laparoscopic removal and scar repair. ${ }^{19}$ Although 3D-MESIA was not applied in this case, the authors were impressed by the protocol.

\section{Table I 3D MESIA}

\begin{tabular}{ll}
\hline S. no & 3D MESIA \\
\hline I & Methotrexate (MTX) \\
2 & Uterine artery embolization (UAE) \\
3 & Sonography directed In situs Aspiration sequential therapy \\
4 & Based on the pregnancy sac three-dimensional (3D) \\
\hline
\end{tabular}

\section{Conclusion}

CSEP presents specific challenges for clinicians limiting a single gold standard treatment procedure. A SILS surgical approach in combination with hysteroscopy has been effective; however, 
incorporation of the 3D-MESIA protocol has shown better treatment options and patient outcomes. The difficulty with managing CSEP places greater emphasis on patient education and recurrence prevention. Patients who desire subsequent pregnancies should be educated about possible complications, as well as management options. For patients who prefer to abstain, the use of long-acting reversible contraception such as Nexplanon subcutaneous implant is the most effective form of post-partum and post-procedure contraception-since it does not require intrauterine placement. ${ }^{19}$

\section{Acknowledgements}

None.

\section{Conflicts of interest}

Dr. Xiaoming Guan is a speaker for Applied Medical, Rancho Santa Margarita, California. Other authors did not report any potential conflicts of interests.

\section{References}

1. Shu SR, Luo X, Wang ZX, et al. Cesarean scar pregnancy treated by curettage and aspiration guided by laparoscopy. Ther Clin Risk Manag. 2015;11:1139-41

2. Rotas MA, Haberman S, Levgur M. Cesarean scar ectopic pregnancies: etiology, diagnosis, and management. Obstet Gynecol. 2006;107(6):1373-81

3. Mahgoub S, Gabriele V, Faller E, et al. Cesarean scar ectopic pregnancy. Laparoscopic resection and total scar dehiscence repair. J Minim Invasive Gynecol. 2017;25(2):297-8.

4. Fylstra DL. Ectopic pregnancy within a cesarean scar: a review. Obstet Gynecol Surv. 2002;57(8):537-43

5. Gonzalez N, Tulandi T. Cesarean Scar Pregnancy: A Systematic Review, J Minim Invasive Gynecol. 2017;24(5):731-38.

6. Nankali A, Ataee M, Shahlazadeh H, et al. Surgical management of the cesarean scar ectopic pregnancy: a case report. Case Rep Obstet Gynecol. 2013:525187.
7. Chukus A, Tirada N, Restrepo R, et al. Uncommon Implantation Sites of Ectopic Pregnancy: Thinking beyond the Complex Adnexal Mass. Radiographics. 2015;35(3):946-59.

8. Ash A, Smith A, Maxwell D. Caesarean scar pregnancy. BJOG. 2007;114(3):253-63.

9. Maymon R, Halperin R, Mendlovic S, et al. Ectopic pregnancies in a Caesarean scar: review of the medical approach to an iatrogenic complication. Hum Reprod Update. 2004;10(6):515-23.

10. Liu S, Durai S. Management of a case of caesarean scar pregnancy and all its complications. BMJ Case Rep. 2016.

11. Seow KM, Huang LW, Lin YH, et al. Cesarean scar pregnancy: issues in management. Ultrasound Obstet Gynecol. 2004;23(3):247-53.

12. Sieczko D, Edwards H, Heggs K, et al. Caesarean scar ectopic pregnancy: a case report. Ultrasound. 2014;22(2):126-8.

13. Hudeček R, Felsingerová Z, Felsinger M, et al. Laparoscopic Treatment of Cesarean Scar Ectopic Pregnancy. J Gynecol Surg. 2014;30(5):309-11.

14. Wang YL, Su TH, Chen HS. Operative laparoscopy for unruptured ectopic pregnancy in a caesarean scar. BJOG. 2006;113(9):1035-8.

15. Maheux-Lacroix S, Li F, Bujold E, et al. Cesarean Scar Pregnancies: A Systematic Review of Treatment Options. J Minim Invasive Gynecol. 2017;24(6):915-25.

16. Litwicka K, Greco E. Caesarean scar pregnancy: a review of management options. Curr Opin Obstet Gynecol. 2011;23(6):415-21.

17. Lee JH, Kim SH, Cho SH, et al. Laparoscopic surgery of ectopic gestational sac implanted in the cesarean section scar. Surg Laparosc Endosc Percutan Tech. 2008;18(5):479-82.

18. Roy MM, Radfar F. Management of a Viable Cesarean Scar Pregnancy: A Case Report. Oman Med J. 2017;32(2):161-66.

19. Wang YJ, Zhai Y, Zhang ZY. Clinical features and outcome of cesarean scar pregnancy. Zhonghua Yi Xue Za Zhi. 2017;97(13):986-90.

20. Hubacher D, Spector H, Monteith C, et al. Long-acting reversible contraceptive acceptability and unintended pregnancy among women presenting for short-acting methods: a randomized patient preference trial. Am J Obstet Gynecol. 2017;216(2):101-9. 